\title{
Complex Jacobi Matrices and Gauss Quadrature for Quasi-definite Linear Functionals
}

\author{
S. Pozza', M. Pranić ${ }^{2}$, and Z. Strakoš³

The Gauss quadrature can be formulated as a method for approximation of positive definite linear functionals. The underlying theory connects several classical topics including orthogonal polynomials and (real) Jacobi matrices. In [4] we investigated the problem of generalizing the concept of Gauss quadrature for approximation of linear functionals which are not positive definite. We showed that the concept can be generalized to quasi-definite functionals and based on a close relationship with orthogonal polynomials and complex Jacobi matrices.

\section{Orthogonal polynomials}

First we recall the basic theory of orthogonal polynomials ([2]) Let $\mathcal{L}: \mathcal{P} \rightarrow \mathbb{C}$ be a linear functional defined on the space of complex polynomials $\mathcal{P}$.

Definition $1 \mathrm{~A}$ sequence of polynomials $p_{0}, p_{1}, \ldots, p_{k}$ such that $\operatorname{deg}\left(p_{j}\right)=j\left(\pi_{j}\right.$ is of degree $\left.j\right), \mathcal{L}\left(p_{i} p_{j}\right)=0, i<j$ and $\mathcal{L}\left(p_{j}^{2}\right) \neq 0$, is called a sequence of orthogonal polynomials with respect to the linear functional $\mathcal{L}$.

We say moments of a linear functional the values $\mathcal{L}\left(x^{i}\right)=m_{i}$ for $i=0,1, \ldots$ We define the Hankel determinants $\Delta_{j}$ of the matrices of moments as

$$
\Delta_{j}=\left|\begin{array}{cccc}
m_{0} & m_{1} & \ldots & m_{j} \\
m_{1} & m_{2} & \ldots & m_{j+1} \\
\vdots & \vdots & \ddots & \vdots \\
m_{j} & m_{j+1} & \ldots & m_{2 j}
\end{array}\right|
$$

Definition 2 A linear functional $\mathcal{L}$ for which the first $k+1$ Hanke determinants are nonzero, i.e., $\Delta_{j} \neq 0$ for $j=0,1, \ldots, k$, is called quasi-definite on the space of polynomials $\mathcal{P}_{k}$ of degree at most $k$.

The linear functional $\mathcal{L}$ is said to be positive definite on $\mathcal{P}_{k}$ if $m_{s} \in \mathbb{R}$ for $s=0, \ldots, 2 k$ and $\Delta_{j}>0$ for $j=0, \ldots, k$. Moreover, there exists a positive non-decreasing distribution function $\mu$ supported on the real axis such that $\mathcal{L}(p)=\int p(x) \mathrm{d} \mu(x)$ for all $p$ from $\mathcal{P}_{2 k}$. Hence, positive definite linear functionals are considered by the classic theory of Gauss quadrature.

Any sequence of orthogonal polynomials $p_{0}, p_{1}, \ldots$ satisfies the three term recurrence relationship (see [2, p. 19]) which can be written in the matrix form

$$
x\left[\begin{array}{c}
p_{0}(x) \\
p_{1}(x) \\
\vdots \\
p_{n-1}(x)
\end{array}\right]=T_{n}\left[\begin{array}{c}
p_{0}(x) \\
p_{1}(x) \\
\vdots \\
p_{n-1}(x)
\end{array}\right]+\beta_{n}\left[\begin{array}{c}
0 \\
0 \\
\vdots \\
p_{n}(x)
\end{array}\right] .
$$

$T_{n}$ is a tridiagonal complex matrix

\section{The eigenvalues of $T_{n}$ are the zeros of $p_{n}$}

If we consider a sequence of orthonormal polynomials, i.e., such that $\mathcal{L}\left(p_{i}^{2}\right)=1$ for $i=0, \ldots, n-1$, then $T_{n}$ is a symmetric matrix.

\section{Jacobi matrices}

We define Jacobi matrix the complex matrix

$$
J_{n}=\left[\begin{array}{cccc}
\alpha_{0} & \beta_{1} & & \\
\beta_{1} & \alpha_{1} & \ldots & \\
& \cdots & \cdots & \beta_{n-1} \\
& & \beta_{n-1} & \alpha_{n-1}
\end{array}\right]
$$

with $\beta_{i} \neq 0$ for $i=1, \ldots, n-1$ (see [1]) only if it has distinct eigenvalues.

We proved the following theorem for the case of a quasi-definite linear functional.

Theorem 2 [Moment Matching Property] Let $\mathcal{L}$ be a quasinomials with respect to $\mathcal{L}$. Then

\section{$\mathcal{L}\left(x^{i}\right)=m_{0} \mathbf{e}_{1}^{T}\left(J_{n}\right)^{i} \mathbf{e}_{1}, \quad i=0, \ldots, 2 n-1$,}

\section{A real $J_{n}$ is diagonalizable and has distinct eigenvalues. \\ If $J_{n}$ is complex we have the following statements. \\ Theorem 1 Every tridiagonal matrix $T \in \mathbb{C}^{n \times n}$ with nonzero ele ments on its super-diagonal (or sub-diagonal) is non-derogatory i.e., its eigenvalues have geometric multiplicity 1 .}

\section{A complex $J_{n}$ is diagonalizable if and} definite linear functional on $\mathcal{P}_{n}$ and let $J_{n}$ be the Jacobi matrix of coefficients from the recurrence relations for orthogonal polywhere $m_{0}=\mathcal{L}\left(x^{0}\right)$.

\section{for $f \in \mathcal{P}_{2 n-1}, \quad \mathcal{L}(f)$
Unicity}

Theorem 5 Let $\mathcal{L}$ be an arbitrary linear functional on $\mathcal{P}$. The $n$-weight quadrature (2) of degree of exactness at least $2 n-1$ exists and is unique if and only if the $n$-th Hankel determinant is nonzero, i.e., $\Delta_{n-1} \neq 0$.

\section{Exactness}

Finally, the following theorem gives the condition under which the degree of exactness of (2) is exactly $2 n-1$ (i.e., it does not exceed $2 n-1$ ). This issue has no counterpart in the positivedefinite case where the $n$-node Gauss quadrature cannot have definite case where the $n$-node Gauss quadrature

\section{Quadrature rule}

Positive definite linear functionals naturally lead to the wellknown Gauss quadrature. Let us recall some of its basic properties:

- G1: The $n$-node Gauss quadrature attains the maximal alge braic degree of exactness $2 n-1$.

-G2: The $n$-node Gauss quadrature is well-defined and it is unique. Naturally, the Gauss quadratures with a smaller number of nodes also exist and they are unique.

- G3: The Gauss quadrature of the function $f$ can be written in the form $m_{0} \mathbf{e}_{1}^{T} f\left(J_{n}\right) \mathbf{e}_{1}$, where $J_{n}$ is the Jacobi matrix containing the coefficients from the three-term recurrence relation for orthonormal polynomials associated with $\mathcal{L} ; m_{0}=\mathcal{L}\left(x^{0}\right)$.

The nodes of the Gauss quadrature are the eigenvalues of $J_{n}$ and the weights are given by interpolation.

In [4] we revisit the situation for the functional $\mathcal{L}$ that is only quasidefinite. We start with the usual form of an $n$-node quadrature

$$
\mathcal{L}(f)=\sum_{i=1}^{n} \omega_{i} f\left(\lambda_{i}\right)+R_{n}(f),
$$

where the nodes $\lambda_{1}, \ldots, \lambda_{n}$ are distinct and the last term stands for the quadrature error.

Theorem 3 The quadrature (1) is exact for every $f$ from $\mathcal{P}_{2 n-1}$ if and only if it is interpolatory and the polynomial

$$
\varphi_{n}(x)=\prod_{i=1}^{n}\left(x-\lambda_{i}\right)
$$

satisfies $\mathcal{L}\left(\varphi_{n} p\right)=0$ for every $p \in \mathcal{P}_{n-1}$

The quadrature rule (1) has the properties $\mathrm{G} 1$ and $\mathrm{G} 2$ if and only

- $\quad \mathcal{L}$ is quasi-definite on $\mathcal{P}_{n}$

- $J_{1}, \ldots, J_{n}$ are diagonalizable

In order to avoid this restriction we modify the quadrature considering the $n$-weight quadrature formula

$$
\mathcal{L}(f)=\sum_{i=1}^{\ell} \sum_{j=0}^{s_{i}-1} \omega_{i, j} f^{(j)}\left(\lambda_{i}\right)+R_{n}(f),
$$

where $n=s_{1}+\ldots+s_{\ell}$

Theorem 4 Let $\mathcal{L}$ be an arbitrary linear functional on $\mathcal{P}$. The quadrature (2) is exact for every $f$ from $\mathcal{P}_{2 n-1}$ if and only if it is exact on $\mathcal{P}_{n-1}$ and the polynomial

$$
\varphi_{n}(x)=\left(x-\lambda_{1}\right)^{s_{1}}\left(x-\lambda_{2}\right)^{s_{2}} \ldots\left(x-\lambda_{\ell}\right)^{s_{\ell}}
$$

satisfies $\mathcal{L}\left(\varphi_{n} p\right)=0$ for every $p \in \mathcal{P}_{n-1}$

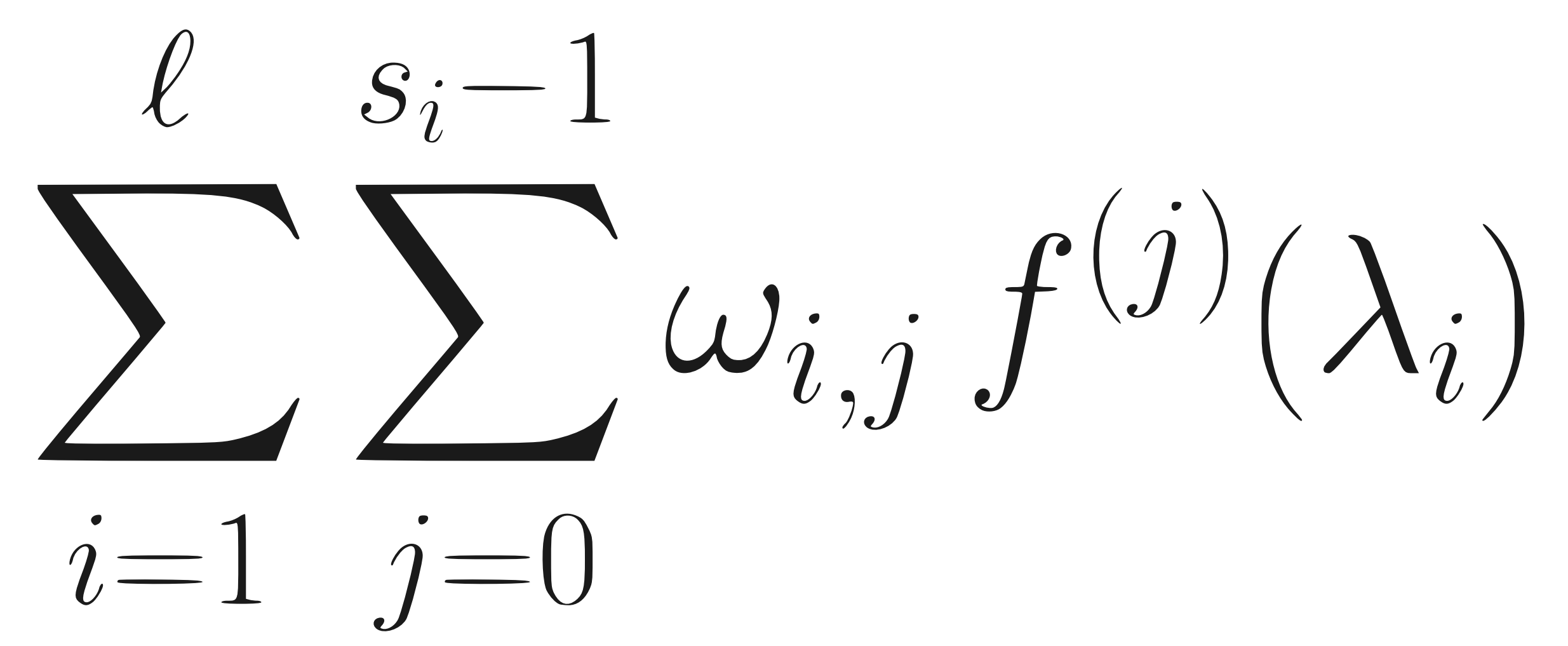

\section{Bibliography}

algebraic degree of exactness larger than $2 n-1$. Theorem 6 Let $\mathcal{L}$ be an arbitrary linear functional on $\mathcal{P}$ and let the $n$-weight quadrature (2) has deg $2 n-1$. Then the degree of exactness of the quadrature (2) is (exactly) $2 n-1$ if and only if the $(n+1)$-st Hankel determinant is nonzero, i.e., $\Delta_{n} \neq 0$.
[1] B. Beckermann, Complex Jacobi matrices, J. Comput. Appl. Math. 127 (2001) 17-65.

[2] T.S. Chihara, An introduction to orthogonal polynomials, Gordon and Breach, New York, 1978.

[3] J. Liesen, Z. Strakoš, Krylov Subspace Methods: Principles and Analysis, Oxford University Press, Oxford, 2013. definite linear functionals, submitted.
[4] S. Pozza, M. Pranić, Z. Strakoš, Gauss quadrature for quasi- 